\title{
Physics in Discrete Spaces: On the Standard Model of Particles
}

\author{
Pierre Peretto \\ Laboratory of Physics and Modelling of Condensed Matter, Grenoble, France \\ Email: Pierre.peretto@lpmmc.cnrs.fr
}

Received 28 January 2015; accepted 23 May 2015; published 26 May 2015

Copyright (C) 2015 by author and Scientific Research Publishing Inc.

This work is licensed under the Creative Commons Attribution International License (CC BY).

http://creativecommons.org/licenses/by/4.0/

c) (†) Open Access

\begin{abstract}
We show that the model of discrete spaces that we have proposed in previous contributions gives a comprehensive and detailed interpretation of the properties of the standard model of particles. Moreover the model also suggests the possible existence of a non-standard family of particles.
\end{abstract}

Keywords

Standard Model of Particles, Chirality, Regge Trajectories, CKM Matrix

\section{Introduction}

According to the model of discrete space-time that we have put forward in preceding contributions, the universe as a whole could be considered as a sort of spin glass that is a set of randomly interacting Ising spins called cosmic bits. This model accounts for several fundamental properties of the physical world, such as the structure of space-time [1], the postulates of quantum theory [2], and the fundamental interactions (gravitation included) [3]. To be convinced that, the model provides a general description of natural phenomena, but something essential is still missing: it must explain the properties of matter itself that is it must provide an interpretation to the standard model of particles. Usual theories of natural phenomena appeal to the concept of mathematical points that are systems deprived of the possibility of housing such complex structures as elementary particles. The model of discrete space-time offers this possibility because, in a discrete space, a physical point, called a world point, has a finite physical size. A discrete space-time model so allows an internal organization to develop inside world points, providing a way for the building of complex structures. This is the object of the present contribution.

\section{The Group $S_{4}$ and the Organization of Elementary Particles}

\subsection{Structure of Elementary Particles in Discrete Spaces}

According to the hypothesis that the universe is discrete, the elementary particles are structures built around

How to cite this paper: Peretto, P. (2015) Physics in Discrete Spaces: On the Standard Model of Particles. Journal of Modern Physics, 6, 811-827. http://dx.doi.org/10.4236/jmp.2015.66086 
world points with a given symmetry. Let us recall that the cosmic bits of a world point are all connected to each other through ferromagnetic interactions [1]. Then space-time disappears inside a world point which therefore seems punctual from an experimental point of view. According to [3] the size $l^{*}$ of a world point would be of the order of $l^{*} \cong 0.5 \times 10^{-21} \mathrm{~cm}$.

Our approach suggests that a particle could be structured along a three levels organization

1) A particle is built around a special world point (whose size is $l^{*}$ ), called its seed. The internal interactions matrix of the seed $G_{P}$ must transform according to gauge symmetries and the symmetry properties of the seed determine the symmetry of the whole particle.

2) The seed modifies the polarization states of the neighbouring world points over a range $\rho l^{*}$. This region of influence is called the core of the particle and the system made of a seed and its core constitutes a bare particle.

3) Finally the bare particle is surrounded by a cloud of gauge particles whose nature and properties are determined by the symmetry of the seed according to the gauge symmetry mechanisms that have been discussed in [3]. The system made of a bare particle and its cloud of virtual particles constitutes a dressed particle.

\subsection{The Irreducible Representations of $S_{4}$}

The permutation group $S_{4}$ of four objects, a finite group, plays a prominent role in our approach as already stressed in [1]-[3]. Physics, indeed, must be invariant under any permutation of the four axes used to describe the internal states $\phi_{i}$ of world point $i$ since no specific orientation of axes can be defined inside a world point. The permutation group $S_{4}$ of four objects has 24 elements distributed into 5 classes. The table of characters of $S_{4}$ may be found in [1]. The internal interactions matrices $G_{P}$ of the seed must commute with any four dimensional representation of group $S_{4}$. Therefore it must transform according to direct sums of irreducible representations of $S_{4}$. There are two types of irreducible representations. On the one hand $\Gamma_{1}$ and $\Gamma_{3}$, that are insensitive to mirror operations that is to an odd number of permutations. On the other $\Gamma_{1}^{*}$ and $\Gamma_{3}^{*}$ are sensitive to mirror operations. Finally $\Gamma_{2}$ does not take the mirror operations into account. We see below that the particles imply the representations $\Gamma_{1}, \Gamma_{2}$ and $\Gamma_{3}$ and the antiparticles the representations $\Gamma_{1}^{*}, \Gamma_{2}^{*} \equiv \Gamma_{2}$ and $\Gamma_{3}^{*}$. The problem of finding four-dimensional matrices $G_{P}$ as direct sums of $\Gamma_{1}, \Gamma_{2}$ and $\Gamma_{3}$ representations has the following solutions.
a) $4=1+3$
b) $4=2+2$
c) $4=1+1+2$
d) $4=1+1+1+1$

The matrices $G_{B}$ that transform along to solution a), that is $G_{B} \cong \Gamma_{1} \oplus \Gamma_{3}$, define the seeds of boson particles. The matrices $G_{F}$ that transform along to solution b), that is $G_{F} \cong \Gamma_{2} \oplus \Gamma_{2}$, define the seeds of fermion particles (see [2]).

\subsection{Recovering the Organization of the Standard Model of Particles}

\subsubsection{Particles}

Super-symmetry theory (Susy) puts forward that fermions and bosons may be considered as two aspects of the very same objects. In a spirit close to that of Susy it is proposed here that the fundamental particles are objects that associate world points with different symmetries. A bare particle would, accordingly, be made of a pair of coupled world points, one undergoing a fermionic polarization and the other a bosonic polarization. The idea that an elementary particle could be formed of a couple of bosonic and fermionic sub-particles has also been suggested by Koide [4] and is implicit, through the $U(1) \times \mathrm{SU}(2)$ symmetry group, in the GSW theory of electroweak interactions [3].

The state of a particle is then represented in a 16-dimensional vector space that is obtained by the direct product of the two 4-dimensional spaces associated with the members of the pair that form the particle. The states must therefore transform as

$$
\left(\Gamma_{1} \oplus \Gamma_{3}\right) \otimes\left(\Gamma_{2} \oplus \Gamma_{2}\right),
$$

that may be expanded along

$$
\left(\Gamma_{1}\right) \otimes\left(\Gamma_{2} \oplus \Gamma_{2}\right) \oplus\left(\Gamma_{3}\right) \otimes\left(\Gamma_{2} \oplus \Gamma_{2}\right) .
$$


There are therefore four particles. The two particles associated with $\left(\Gamma_{1}\right) \otimes\left(\Gamma_{2} \oplus \Gamma_{2}\right)=\left(\Gamma_{1} \otimes \Gamma_{2}\right) \oplus\left(\Gamma_{1} \otimes \Gamma_{2}\right)$ are called leptons. The two particles associated with $\left(\Gamma_{3}\right) \otimes\left(\Gamma_{2} \oplus \Gamma_{2}\right)=\left(\Gamma_{3} \otimes \Gamma_{2}\right) \oplus\left(\Gamma_{3} \otimes \Gamma_{2}\right)$ are called quarks. Since all these particles transform along $\Gamma_{2}$ they are all fermions.

\subsubsection{Antiparticles}

We now consider how antiparticles may be derived from the same formalism. The different types of particles have been determined by using the mirror insensitive representations $\Gamma_{1} \Gamma_{2}$ and $\Gamma_{3}$. The types of anti- particles are determined by using the mirror sensitive representations $\Gamma_{1}^{*}, \Gamma_{3}^{*}=\Gamma_{1}^{*} \otimes \Gamma_{3}$ and $\Gamma_{2}^{*}=\Gamma_{1}^{*} \otimes \Gamma_{2}=\Gamma_{2}$. Since $\Gamma_{1}^{*}$ and $\Gamma_{3}^{*}$ are sensitive to mirror operations the state $\bar{\phi}$ associated with an anti-particle is obtained from the state $\phi$ associated with a particle through a mirror transformation that is a sign change of one of its components. Nothing, however, determines which component has to be modified and all signs have to be changed at once that is

$$
\bar{\phi}=\left(\begin{array}{l}
-\varphi_{1} \\
-\varphi_{2} \\
-\varphi_{3} \\
-\varphi_{4}
\end{array}\right)=\left(\begin{array}{llll}
-1 & & & \\
& -1 & & \\
& & -1 & \\
& & & -1
\end{array}\right)\left(\begin{array}{l}
\varphi_{1} \\
\varphi_{2} \\
\varphi_{3} \\
\varphi_{4}
\end{array}\right)=C \phi .
$$

$C$ is the charge conjugation operator.

Let us remember that the polarization $\phi$ of a world point obeys a mean field equation [1]

$$
\phi=\tanh (b J \phi) .
$$

$J$ is the amplitude of binary interactions between the $n$ cosmic bits of a world point and $b$ is a parameter that materializes a cosmic disorder ( $b^{-1}$ is somehow similar to a cosmic temperature though completely different in nature).

If $\phi$ is a solution of this equation, $-\phi$ is also a solution.

The states of anti-particles then transform as

$$
\left(\Gamma_{1}^{*} \oplus \Gamma_{3}^{*}\right) \otimes\left(\Gamma_{2} \oplus \Gamma_{2}\right),
$$

that may be expanded along

$$
\left(\Gamma_{1}^{*}\right) \otimes\left(\Gamma_{2} \oplus \Gamma_{2}\right) \oplus\left(\Gamma_{3}^{*}\right) \otimes\left(\Gamma_{2} \oplus \Gamma_{2}\right) .
$$

Antiparticles are therefore organized exactly as particles. They have exactly the same properties as the corresponding particles, except for the sign of electric charges which is sensitive to charge conjugation.

We have so far obtained a description of the organization of one family of elementary particles that exactly fits the standard model of particles. However there are three families of particles with identical properties except for their masses. The symmetry properties are the same for the three families and one must admit, accordingly, that the matrices $G_{P}$ are the same for the three families. However while the particles of the first family are stable the particles belonging to the other families are unstable. Therefore, the existence of these families cannot be looked for in some new type of symmetry similar to SU(3) for example. An alternative interpretation is proposed below.

\subsubsection{A Non-Standard Family of Particles}

According to solution (c): $(4=1+1+2)$ the internal interactions matrix would transform according to $G_{n s} \approx \Gamma_{1} \oplus \Gamma_{1} \oplus \Gamma_{2}$, where "ns" is for non-standard.

The particles that possibly emerge from this representation have been ignored so far. We consider that $G_{n s}$ comes from a symmetry breaking $\Gamma_{2} \rightarrow \Gamma_{1} \oplus \Gamma_{1}$. Then a family associated with the representation $\left[\Gamma_{1} \oplus \Gamma_{1} \oplus \Gamma_{2}\right] \otimes\left[\Gamma_{1} \oplus \Gamma_{3}\right]$ can be generated. This gives rise to a family comprised of six particles. We find four bosons:

- $\left(\Gamma_{1} \otimes \Gamma_{1}\right) \oplus\left(\Gamma_{1} \otimes \Gamma_{1}\right)$ : two scalar bosons;

- $\left(\Gamma_{1} \otimes \Gamma_{3}\right) \oplus\left(\Gamma_{1} \otimes \Gamma_{3}\right):$ two vector bosons; 
and two fermions:

- $\left(\Gamma_{2} \otimes \Gamma_{1}\right)$ : one lepton;

- $\left(\Gamma_{2} \otimes \Gamma_{3}\right)$ : one quark.

It is possible that one of these particles corresponds to the particle recently observed at the Large Hadron Collider (LHC). To understand which particle is really at stake we must, however, gain much more information regarding the properties of the found particle.

The two scalar bosons, if they do exist, behave as Higgs scalar particles and could be involved in the same sorts of reactions. If a Higgs boson is defined as a scalar boson the proposed particles are Higgs bosons. If a Higgs boson is defined as a particle that gives masses to otherwise zero mass particles the two particles are not Higgs bosons because, as we will see in the present approach, no Higgs mechanism is necessary to give masses to particles. The lepton could give rise to a new type of neutrino. There are experimental evidences for the existence of a new type of neutrino, called a sterile neutrino, whose (still controversial) existence has possibly been revealed in LSND [5] or Mini-Boone experiments [6].

Finally we can ignore the representation $\Gamma_{1} \oplus \Gamma_{1} \oplus \Gamma_{1} \oplus \Gamma_{1}$ which seems not to have any physical consequences because the so generated space has four time-like and no space-like dimensions.

\section{On Particles Masses}

\subsection{General Considerations}

Let us recall that the state $\psi_{P}$ of a physical system containing a particle $P$ obeys the following eigenvalue equation

$$
\left(\Delta \otimes\{G\}_{P}\right) \psi=\kappa_{P} \psi,
$$

The mass $m_{P}$ of $P$ is given by $m_{P}=\frac{1}{C}\left(\kappa_{P}\right)^{1 / 2}$.

This way of introducing particle masses seems to go against the usual conclusion that all particles must have zero masses due to the dichotomy of the universe between a right $(R)$ and a left $(L)$ universe. The well known argument goes as follows:

The mass term in the Lagrangian of a fermion field $\psi$ writes

$$
m \psi^{C} \psi=m \psi^{\mathrm{T}} \gamma^{1} \psi=m\left(\psi_{R}^{C}+\psi_{L}^{C}\right)\left(\psi_{R}+\psi_{L}\right) .
$$

We consider the right handed term $\psi_{R}^{C} \psi_{R}$. We define a projection operator $\left(1+\gamma^{5}\right) / 2$ with $\gamma^{5}=\mathrm{i} \gamma^{1} \gamma^{2} \gamma^{3} \gamma^{4}$ where $\left(\gamma^{1}, \gamma^{2}, \gamma^{3}, \gamma^{4}\right)$ are the four Dirac matrices. The projection operator transforms a general state $\psi$ into a right handed state $\psi_{R}$

$$
\psi_{R}=\frac{\left(1+\gamma^{5}\right)}{2} \psi
$$

Since $\gamma^{1} \gamma^{5}=-\gamma^{5} \gamma^{1}$ one also has

$$
\psi_{R}^{C}=\psi_{R}^{C} \frac{\left(1-\gamma^{5}\right)}{2},
$$

and thus

$$
\psi_{R}^{C} \psi_{R}=\frac{1}{4} \psi^{C}\left(1-\gamma^{5}\right)\left(1+\gamma^{5}\right) \psi=\frac{1}{4} \psi^{C}\left(1-\left(\gamma^{5}\right)^{2}\right) \psi=0 .
$$

Likewise $\psi_{L}^{C} \psi_{L}=0$. All other terms, such as $\psi_{R}^{C} \psi_{L}$, mix the chiralities and are therefore forbidden. Consequently there are no more mass terms left in the Lagrangian.

If there is no universe dichotomy this derivation no longer holds and the particles may have non zero-masses.

The universe dichotomy is introduced to account for the experimental observation that the neutrinos are left handed. We show in Section 4.1 that this property of neutrinos is, in reality, a consequence of the model and no 
Higgs mechanism is needed to give a mass to particles. It must be stressed, however, that a Higgs scalar field, identified with the world point polarization state $\left|\phi_{i}\right|$, is still necessary simply to give an existence to particles.

\subsection{Seeds Masses}

The states $\phi_{P}^{\text {seed }}$ of an isolated seed, associated with a particle $P$, are obtained by minimizing the Lagrangian

$$
\Lambda_{P}^{\text {seed }}\left(\phi_{P}^{\text {seed }}\right)=\phi_{P}^{\text {seed, }, T} G_{P} \phi_{P}^{\text {seed }},
$$

under the constraint $\phi_{P}^{\text {seed,T }} \phi_{P}^{\text {seed }}=1$. The states of the seed thus satisfy the following eigenvalue equation

$$
G_{P} \phi_{P}^{\text {seed }}=\kappa_{P}^{\text {seed }} \phi_{P}^{\text {seed }} .
$$

The mass $m_{P}^{\text {seed }}$ of the seed is given by

$$
m_{P}^{\text {seed }}=\frac{\hbar}{C}\left(\kappa_{P}^{\text {seed }}\right)^{1 / 2}
$$

\subsubsection{Bosonic Seeds}

Since the internal interaction matrix $G_{B}$ of bosonic seeds must transform according to the direct sum $\left(\Gamma_{1} \oplus \Gamma_{3}\right)$, its diagonal form writes

$$
G_{B}=\left(\begin{array}{llll}
\kappa_{B 1} & & & \\
& \kappa_{B 2} & & \\
& & \kappa_{B 2} & \\
& & & \kappa_{B 2}
\end{array}\right) .
$$

The analytical form of matrix $G_{B}$ may be found from the dynamics of boson fields that is from the KleinGordon equation

$$
\left(-\frac{1}{c^{2}} \frac{\partial^{2}}{\partial t^{2}}+\Delta+\right) \psi_{v}(r, t)=\left(\frac{m c}{\hbar}\right)^{2} \psi_{v}(r, t)
$$

by isolating a world point $i$ from its neighbourhood. This is achieved by replacing the differential operators $\partial_{\mu}^{2}$ by Dirac functions $\partial_{\mu}^{2} \rightarrow \delta\left(x_{\mu}\right)$ with $\delta\left(x_{\mu}\right)=1$ if $x_{\mu}=0$ and $\delta\left(x_{\mu}\right)=0$ otherwise. $G_{B}$ is then given by

$$
G_{B}=\left(\begin{array}{cccc}
-1 / c^{2} & & & \\
& 1 & & \\
& & 1 & \\
& & & 1
\end{array}\right) .
$$

$G_{B}$, therefore, is proportional to the metric matrix of vacuum $G^{\text {vacuum }}$. The eigenvalues of $G_{B}$ are $\kappa_{B 1}=-1 / c^{2}$, a non degenerate time-like eigenvalue and $\kappa_{B 2}=1$, a three fold degenerate space-like eigenvalue.

\subsubsection{Fermionic Seeds}

Since the internal interaction matrix $G_{F}$ of fermionic seeds must transform according to the direct sum $\left(\Gamma_{2} \oplus \Gamma_{2}\right)$, its diagonal form writes

$$
G_{F}=\left(\begin{array}{cccc}
\kappa_{F 1} & & & \\
& \kappa_{F 1} & & \\
& & \kappa_{F 2} & \\
& & & \kappa_{F 2}
\end{array}\right) .
$$

The analytical form of matrix $G_{F}$ may be found from the equation of fermion fields that is from the Dirac equation 


$$
\left(\sum_{\mu} \mathrm{i} \hbar \gamma^{\mu} \partial_{\mu}-m c\right) \psi=0
$$

by isolating a world point $i$ from its neighbourhood. Then

$$
G_{F}=\left[\frac{\gamma^{1}}{c}+\gamma^{2}+\gamma^{3}+\gamma^{4}\right] .
$$

The Dirac matrices $\gamma^{\mu}$ are expressed in a basis (the Dirac representation) where the indices $\mu$ of matrices $\gamma^{\mu}$ are identified with the Lorentz indices $\mu$ of space-time itself. The matrix $\gamma^{1}$

$$
\gamma^{1}=\left(\begin{array}{llll}
1 & & & \\
& 1 & & \\
& & -1 & \\
& & & -1
\end{array}\right)
$$

is introduced so as to make hermitian the operator $\Lambda_{F}$. Then

$$
G_{F}=\gamma^{1}\left(\frac{1}{c} \gamma^{1}+\gamma^{2}+\gamma^{3}+\gamma^{4}\right)
$$

and the matrix $G_{F}$ finally writes

$$
G_{F}=\left(\begin{array}{cccc}
1 / c & 0 & 1 & 1+\mathrm{i} \\
0 & 1 / c & 1-\mathrm{i} & -1 \\
1 & 1+\mathrm{i} & 1 / c & 0 \\
1-\mathrm{i} & -1 & 0 & 1 / c
\end{array}\right) .
$$

As expected $G_{F}$ has two real two-fold degenerate eigenvalues $\kappa_{F 1}=(1 / c-\sqrt{3})$ and $\kappa_{F 2}=(1 / c+\sqrt{3})$.

The lepton associated with the eigenvalue $\kappa_{F 2}=(1 / c+\sqrt{3})$ is called the neutrino, that associated with $\kappa_{F 1}=(1 / c-\sqrt{3})$ is called the electron. The quark associated with eigenvalue $\kappa_{F 2}=(1 / c+\sqrt{3})$ is called the down quark $d$ and, finally, the quark associated with eigenvalue $\kappa_{F 1}=(1 / c-\sqrt{3})$ is called the up quark $u$. The representation $\Gamma_{3}$ introduces a three-fold degeneracy, associated with colour quantum number, for the states of quarks.

\subsection{Masses of Bare Particles}

By using a first order perturbation expansion we find that the eigenvalue $\kappa_{P}^{b}$ of a bare particle associated with a seed $P$ is simply proportional to the eigenvalue (here and in the following sections $s$ or seed will be used for seed, $d$ or dressed for dressed, bare for bare and finally $v b$ or virtual bosons for virtual bosons).

Proof:

A bare particle, we have seen in Section 2.1, is comprised of a seed and its core. Let $i$ be the label of world points belonging to the core. The vacuum states $\phi_{i}^{\text {vacuum }}$ of world points belonging to the core are modified by the state $\phi_{P}^{\text {sed }}$ of the seed. To first order they become $\phi_{i}^{\text {vacuum }} \Rightarrow \phi_{i}^{\text {vacuum }}+\varepsilon_{i} \phi_{P}^{\text {seed }}$. Similarly the states modifications in the core induce a reorganization of the cosmic bits of world points $i$, and, therefore of the internal interaction matrices. $G_{i}$ becomes $G_{i}^{\text {vacuum }} \Rightarrow G_{i}^{\text {vacuum }}+\delta_{i} G_{P}$.

The eigenvalue $\kappa_{P}^{\text {bare }}$ of the bare particle then writes

$$
\kappa_{P}^{\text {bare }}=\sum_{i}\left(\phi_{i}^{\text {vacuum }}+\varepsilon_{i} \phi_{P}^{\text {seed }}\right)^{\mathrm{T}}\left(G_{i}^{\text {vacuum }}+\delta_{i} G_{P}\right)\left(\phi_{i}^{\text {vacuum }}+\varepsilon_{i} \phi_{P}^{\text {seed }}\right) .
$$
tain

There is no orientation correlations between $\phi_{i}^{\text {vacuum }}$ and $\phi_{P}^{\text {sed }}$. Therefore, the cross terms vanish and we ob-

$$
\kappa_{P}^{\mathrm{bare}}=\sum_{i} \varepsilon_{i} \phi_{P}^{\mathrm{seed}, \mathrm{T}} \delta_{i} G_{P} \varepsilon_{i} \phi_{P}^{\mathrm{seedT}}=\sum_{i} \varepsilon_{i}^{2} \delta_{i} \kappa_{P}^{\mathrm{seed}}=K \kappa_{P}^{\text {seed }}
$$




\subsection{Analytical Expressions for Bare Particles Masses}

The Lagrangian operator of a particle, that is of a coupled pair of world points, one of bosonic type, the other of fermionic type, must keep the symmetry properties of the two components taken separately so as to preserve the organization of the particles of the Standard Model. This compels the Lagrangian to be a 16-dimensional operator given by

$$
\Lambda_{P}=G_{B} \otimes 1^{(4)}+1^{(4)} \otimes G_{F}+\zeta G_{B} \otimes G_{F}
$$

$\mathrm{I}^{(4)}$ is the four dimensional unit matrix. The coupling parameter $\zeta$ is determined by the organization of interactions between the two world points that constitute a particle, more precisely by the relative numbers $n_{+}$and $n_{-}$of ferromagnetic and antiferromagnetic interactions that link the two points. For example $\zeta=$ $\left(n_{+}-n_{-}\right) /\left(n_{+}+n_{-}\right)$. We have no indication as regards an evaluation of the coupling parameter $\zeta$ except that the values $\zeta=0$ and $\zeta=1$ are forbidden, $\zeta=0$ because the two world points that form the particle would not be coupled and $\zeta=1$ because the coupling would be so strong that the two world points would be in the same state. A value $\zeta=0.5$ could be a reasonable guess but we will see that the results are, in actual fact, weakly $\zeta$ dependent.

The Lagrangian (4) has four eigenvalues. The first is associated with the bare down quark

$$
\kappa_{\text {down }}^{\text {bare }}=\kappa_{B 2}+\kappa_{F 2}+\zeta \kappa_{B 2} \kappa_{F 2}
$$

The second is associated with the bare up quark

$$
\kappa_{\text {up }}^{\text {bare }}=\kappa_{B 2}+\kappa_{F 1}+\zeta \kappa_{B 2} \kappa_{F 1}
$$

That is

$$
\kappa_{\mathrm{down}}^{\mathrm{bare}}=1+\left(\frac{1}{c}+\sqrt{3}\right)+\zeta\left(\frac{1}{c}+\sqrt{3}\right)
$$

and

$$
\kappa_{\text {up }}^{\mathrm{bare}}=1+\left(\frac{1}{c}-\sqrt{3}\right)+\zeta\left(\frac{1}{c}-\sqrt{3}\right)
$$

Since the masses of bare particles are proportional to the masses of their seeds one has

$$
m_{\text {up }}^{\text {bare }} / m_{\text {down }}^{\text {bare }}=\left(\kappa_{\text {up }}^{\text {bare }} / \kappa_{\text {down }}^{\text {bare }}\right)^{1 / 2}=\left(\frac{1+\left(\frac{1}{c}-\sqrt{3}\right)+\zeta\left(\frac{1}{c}-\sqrt{3}\right)}{1+\left(\frac{1}{c}+\sqrt{3}\right)+\zeta\left(\frac{1}{c}+\sqrt{3}\right)}\right)^{1 / 2}
$$

For leptons, however, no calculation of bare particles has been carried out. It is then necessary to add the effects of virtual bosons. Then to calculate their dressed masses one must write

$$
\kappa_{v}^{\text {dressed }}=\kappa_{B 1}+\kappa_{F 1}+\zeta \kappa_{B 1} \kappa_{F 1}+\kappa_{v}^{\nu b}
$$

for the neutrino $v$ and

$$
\kappa_{e l}^{\mathrm{dressed}}=\kappa_{B 1}+\kappa_{F 2}+\zeta \kappa_{B 1} \kappa_{F 2}+\kappa_{e l}^{\nu b}
$$

for the electron. Explicitly one has

$$
\kappa_{v}^{\text {dressed }}=-\frac{1}{c^{2}}+\left(\frac{1}{c}+\sqrt{3}\right)-\frac{1}{c^{2}} \zeta\left(\frac{1}{c}+\sqrt{3}\right)+\kappa_{v}^{\vee b}
$$

and

$$
\kappa_{e l}^{\mathrm{dressed}}=-\frac{1}{c^{2}}+\left(\frac{1}{c}-\sqrt{3}\right)-\frac{1}{c^{2}} \zeta\left(\frac{1}{c}-\sqrt{3}\right)+\kappa_{e l}^{\vee b} .
$$




\subsection{The Zitterbewegung}

The definition of the bare mass $m_{P}^{\text {bare }}$ implies that $\kappa_{P}^{\text {bare }}>0$. If $\kappa_{P}^{\text {bare }}<0$ one has a problem since the mass of the bare particle becomes imaginary. If one wants to keep the minimization principle, and to give nevertheless a physical meaning to negative eigenvalues, one is forced to interpret the negative eigenvalues as zero mass (or almost zero mass) particles. To make this point more precise we consider the set of states $\phi_{\omega}$ defined by $\Lambda_{P}\left(\phi_{\omega}\right)=0$. This set forms a surface (a manifold) $\Sigma_{\omega}$ in the internal space of the world points that generates the bare particle $P$. During the minimization process the state $\phi_{P}$ evolves so as to minimize $\Lambda_{P}\left(\phi_{P}\right)$ under the constraint $\phi_{P}^{\mathrm{T}} \phi_{P}=1$. The trajectory of $\phi_{P}$ possibly crosses $\Sigma_{\omega}$ at a point $\phi_{P}^{\omega}$. Let us then consider a state $\phi_{P}=\phi_{P}^{\omega}+d \phi_{P}$. One has

$$
\Lambda_{P}\left(\phi_{P}\right) \cong \Lambda_{P}\left(\phi_{P}^{\omega}\right)+\left.\nabla \Lambda_{P}\right|_{\phi_{P}=\phi_{P}^{\omega}} d \phi_{P}=\left.\nabla \Lambda_{P}\right|_{\phi_{P}=\phi_{P}^{\omega}} d \phi_{P} .
$$

$\phi_{P}$ has a physical meaning if $m^{2}>0$ that is if $\left.\nabla \Lambda_{P}\right|_{\phi_{P}=\phi_{P}^{\omega}} d \phi_{P}>0$. Crossing $\Sigma_{\omega}$, however, induces a sign change of $\left.\nabla \Lambda_{P}\right|_{\phi_{\mathrm{p}}=\phi_{P}^{\omega}} d \phi_{P}$. If $\phi_{P}$ has a physical meaning in one side of $\Sigma_{\omega}$ it loses this meaning on the other side. To a trajectory of state $\phi_{P}$, however, there corresponds a mirror trajectory of the anti-state $\overline{\phi_{P}}$. On this trajectory the sign of $\left.\nabla \Lambda_{P}\right|_{\phi_{P}=\phi_{P}^{\omega}}$ is changed. Therefore while crossing $\Sigma_{\omega}$, a state $\phi_{P}$, attracted by $\Sigma_{\omega}$, keeps a physical meaning if it transforms into its anti-state $\overline{\phi_{P}}$ which is also attracted by $\Sigma_{\omega}$. This mechanism reminds the Zitterbewegung process, an oscillation between a particle and its antiparticle. The trajectory of $\phi_{P}$ therefore stops on, or remains close to, $\Sigma_{\omega}$. Since $\Lambda_{P}\left(\phi_{P}^{\omega}\right) \cong 0$ the state $\phi_{P}=\phi_{P}^{\omega}$ corresponds to zero, or almost zero mass particles.

The mass of a dressed particle $\kappa_{P}^{\text {dressed }}$ is given by $\kappa_{P}^{\text {dressed }}=\kappa_{P}^{\text {bare }}+\kappa_{P}^{\text {vb }}$. If $\kappa_{P}^{\text {bare }}<0$ while $\kappa_{P}^{\text {dressed }}$ is positive, the mass $m_{P}^{\text {dressed }}$ of the dressed particle keeps a physical meaning which, in the framework of the present model, seems to be the case for the electron (but not for the neutrino). Otherwise the electron would have an imaginary mass which is possibly the reason that makes impossible a calculation of the electron bare mass.

\subsection{Numerical Calculations and Comparison with Experimental Observations}

There is no direct measurement of quark bare masses because it is not possible to observe separate quarks due to their confinement properties. The value of separate bare quark masses can only be obtained through various theoretical calculations that usually lead to different numerical values. The results for quarks bare masses are rather scattered. For example it has been proposed [7] that

$$
3 \mathrm{MeV}<m_{\mathrm{up}}^{\text {bare }}<8 \mathrm{MeV} \text { and } 5 \mathrm{MeV}<m_{\text {down }}^{\text {bare }}<10 \mathrm{MeV}
$$

Calculations, carried out on lattices by C. Davies et al. [8] (see also [9]) give values for the bare masses of light quarks that, the authors claim, are much more accurate than the previously published data. They find

$$
m_{\text {up }}^{\text {bare }}=2.01 \pm 0.14 \mathrm{MeV} \text { and } m_{\text {down }}^{\text {bare }}=4.79 \pm 0.16 \mathrm{MeV}
$$

which gives $m_{\mathrm{up}}^{\text {bare }} / m_{\mathrm{down}}^{\text {bare }}=0.420 \pm 0.04$.

The ratio between bare quark masses is better determined than their explicit values and this is the only value that one can calculate in the present theory without appealing to free parameters. There are two parameters in eq. (9): $c$ and $\zeta$. The constant $c$, the dimensionless speed of light, may be determined through considerations relating to electroweak interactions given in contribution [3]. Explicitly $c=\operatorname{tg}\left(\theta_{W}\right)$ where $\theta_{W}$ is the Weinberg angle. Then $1 / c^{2}=\left[1 / \sin ^{2}\left(\theta_{W}\right)\right]-1$. With the experimental value $\sin ^{2}\left(\theta_{W}\right)=0.231$ this yields $1 / c=1.824$. From the above formula (Equation (9)) one has

$$
m_{\mathrm{up}}^{\text {bare }} / m_{\mathrm{down}}^{\text {bare }}=\left(\frac{1+\left(\frac{1}{c}-\sqrt{3}\right)+\zeta\left(\frac{1}{c}-\sqrt{3}\right)}{1+\left(\frac{1}{c}+\sqrt{3}\right)+\zeta\left(\frac{1}{c}+\sqrt{3}\right)}\right)^{1 / 2}=\left(\frac{1.093+0.093 \zeta}{4.557+3.557 \zeta}\right)^{1 / 2} .
$$

With the value $\zeta=0.5$ (that is $n_{+} / n_{-}=3$ ) one finds $m_{\text {up }}^{b} / m_{\text {down }}^{b}=0.424$, that fits the Davies calculation $m_{\text {up }}^{\text {bare }} / m_{\text {down }}^{\text {bare }}=0.420$ within a $1 \%$ error. 
The range of values given by Davies calculation is $0.38<m_{\text {up }}^{\text {bare }} / m_{\text {down }}^{\text {bare }}<0.46$ and corresponds to the range $0.8>\zeta>0.2$.

The average value $m_{\mathrm{up}}^{\text {bare }} / m_{\mathrm{down}}^{\text {bare }}=0.420$ of Davies is obtained for $\zeta=0.535$ close to the proposed value $\zeta=0.5$.

With the values adopted for $c$ and $\zeta$ one has $\kappa_{v}^{\text {dressed }}=-5.690+\kappa_{v}^{v b}$ and $\kappa_{e l}^{\text {dressed }}=-3.442+\kappa_{e l}^{v b}$. Note that $\kappa_{e l}^{v b}$ must be positive and large enough for $\kappa_{e l}^{\text {dressed }}$ to be positive. Since $\kappa_{e l}^{v b}>\kappa_{v}^{v b}$ (the neutrino is neutral) one has $\kappa_{v}^{\text {dressed }}=-5.690+\kappa_{v}^{v b}<0$. The mass of the neutrino therefore vanishes or, at most, remains very close to zero. In our interpretation the state of a neutrino associated with negative eigenvalues oscillates from one side of the manifold $\Sigma_{\omega}$ to the other side and from the particle to its antiparticle. The mass of a neutrino is the mass it has in the positive side of $\Sigma_{\omega}$. Since the particle must never go far away from $\Sigma_{\omega}$ its mass is very small.

\section{Other Considerations}

\subsection{The Neutrino Left Handed Chirality}

The discovery (by Wu) that the parity symmetry is completely broken by weak interactions and that the electronic neutrino is left handed compelled the physicists to assume that the universe is, in actual fact, made of two different sorts of universes: a left-handed universe $L$ and a right-handed universe $R$. This hypothesis, we have seen, leads to the conclusion that all fermions are zero-mass particles. The eigenvalue associated with neutrinos is negative $\boldsymbol{\kappa}_{v}^{\text {dressed }}<0$ and the state $\phi_{v}$ of a neutrino must stay on or remain in the close vicinity of the manifold $\Sigma_{\omega}$, a surface on which the neutrino state can move freely until the Lagrangian $\Lambda\left(\phi_{v}\right)=\phi_{v}^{\mathrm{T}} G_{F} \phi_{v}$ is minimized, that is until the neutrino state $\phi_{v}$ is parallel to a relevant eigenvector of $G_{F}$. $G_{F}$ can be written as

$$
G_{F}=\frac{1}{C} I^{(4)}+\overline{G_{F}}=\frac{1}{C} I^{(4)}+\left(\begin{array}{cc}
0 & \sigma_{F} \\
\sigma_{F} & 0
\end{array}\right)
$$

with

$$
\sigma_{F}=\left(\begin{array}{cc}
1 & 1+\mathrm{i} \\
1-\mathrm{i} & -1
\end{array}\right)
$$

$\left(\mathrm{i}^{2}=-1\right)$. The eigenstates of $G_{F}$ and those of $\overline{G_{F}}$ are identical. $\overline{G_{F}}$ has two eigenvalues. The eigenvalue $\sqrt{3}$ is associated with the neutrino and the eigenvalue $-\sqrt{3}$ is associated with the electron. Let us write the eigenstate of a neutrino as

$$
\phi_{v}=\left(\begin{array}{l}
\varphi_{1} \\
\varphi_{2} \\
\varphi_{3} \\
\varphi_{4}
\end{array}\right)=\left(\begin{array}{l}
\phi_{1} \\
\phi_{2}
\end{array}\right) .
$$

For $\phi_{v}$ to be an eigenstate of $\overline{G_{F}}$ it is necessary for $\phi_{1}$ and $\phi_{2}$ to be eigenstates of $\sigma_{F}$ with the same eigenvalue $+\sqrt{3}$, (corresponding to neutrinos), and $\phi_{1}$ and $\phi_{2}$ must be identical to one another within a phase factor $\exp (\mathrm{i} \eta)$ whence

$$
\phi_{v}=\left(\begin{array}{c}
\phi_{1} \\
\exp (\mathrm{i} \eta) \phi_{1}
\end{array}\right)
$$

The Lagrangian of a neutrino then writes

$$
\Lambda\left(\phi_{v}\right)=\phi_{v}^{\mathrm{T}} \overline{G_{F}} \phi_{v}=\left[\left(\phi_{1}^{\mathrm{T}}, \exp (-\mathrm{i} \eta) \phi_{1}^{\mathrm{T}}\right)\left(\begin{array}{cc}
0 & I^{(2)} \sqrt{3} \\
I^{(2)} \sqrt{3} & 0
\end{array}\right)\left(\begin{array}{c}
\phi_{1} \\
\exp (\mathrm{i} \eta) \phi_{1}
\end{array}\right)\right]
$$

that is

$$
\Lambda\left(\phi_{v}\right)=\sqrt{3}(\exp (\mathrm{i} \eta)+\exp (-\mathrm{i} \eta))=2 \sqrt{3} \cos (\eta)
$$


$\Lambda\left(\phi_{v}\right)$ is minimum for $\eta=\pi: \Lambda\left(\phi_{v}\right)=-2 \sqrt{3}$ and the eigenstate $\phi_{v}$ of a neutrino writes

$$
\phi_{v}=\left(\begin{array}{c}
\varphi_{1} \\
\varphi_{2} \\
-\varphi_{1} \\
-\varphi_{2}
\end{array}\right)
$$

$\phi_{v}$ is an eigenvector of matrix $\gamma^{5}$ with eigenvalue -1 since

$$
\gamma^{5} \phi_{v}=\left(\begin{array}{cccc} 
& & 1 & \\
& & & 1 \\
1 & & & \\
& 1 & &
\end{array}\right)\left(\begin{array}{c}
\varphi_{1} \\
\varphi_{2} \\
-\varphi_{1} \\
-\varphi_{2}
\end{array}\right)=\left(\begin{array}{c}
-\varphi_{1} \\
-\varphi_{2} \\
\varphi_{1} \\
\varphi_{2}
\end{array}\right)=-\left(\begin{array}{c}
\varphi_{1} \\
\varphi_{2} \\
-\varphi_{1} \\
-\varphi_{2}
\end{array}\right)=-\phi_{v}
$$

The eigenvalue associated with $\phi_{v}$, an eigenvector of $\gamma^{5}$, is negative and the neutrino is, therefore, left-handed. In our approach the neutrino's left handed chirality is a consequence of the general properties of the universe which does not need to be shared into left handed, $L$, and right handed, $R$, universes. The same argument does not hold for the electronic state because $\kappa_{e}^{\text {dressed }}>0$ and the electron state trajectory does not cross the manifold $\Sigma_{\omega}$. Then the electron state is an eigenstate corresponding to the eigenvalue $\kappa_{e}^{\text {dressed }}$ which has no defined chirality.

The particles masses can be computed without appealing to a Higgs mechanism as we have seen. We have already said that the Higgs field, identified with the polarization amplitude $\varphi_{i}\left(\varphi_{i}=\left|\phi_{i}\right|\right)$ of world point $i$, is still necessary, not to give a mass to particles, but simply to give them an existence. The Higgs field is also necessary to make sure that the photon mass is strictly zero as found by the GWS approach of electroweak interactions [3].

The model can however yield a value for the mass $m_{H}$ of Higgs particles if any. The polarization $\varphi$ minimizes the Landau expression (see [1])

$$
F(\varphi)=\lambda \varphi^{2}+\mu \varphi^{4},
$$

where

$$
\lambda=\frac{n(1-b J)}{2 b}, \quad \mu=\frac{n}{12 b} .
$$

The minimum $\varphi_{0}$ of $F$ is given by $\varphi_{0}^{2}=-\lambda / 2 \mu$. We expand $F$ to second order around this minimum

$$
F \cong-\lambda^{2} / 4 \mu-2 \lambda\left(\varphi-\varphi_{0}\right)^{2}
$$

that is

$$
\left(m_{H} / c\right)^{2}=-2 \lambda=\frac{n(b J-1)}{b} .
$$

In other respect one has $\hbar=l^{*}(n / 2 b)^{1 / 2}$ and therefore

$$
m_{H}=\frac{c \hbar}{l^{*}}(2(b J-1))^{1 / 2} .
$$

We now give to $c$ its physical value. With $l^{*} / c=t^{*}=1.6 \times 10^{-31} \mathrm{~s}, \quad b J=4.33$ and $\hbar=6.6 \times 10^{-16} \mathrm{eV} \cdot \mathrm{s}$ one finds

$$
m_{H} \cong 1.21 \times 10^{4} \mathrm{TeV} / \mathrm{c}^{2},
$$

a value that is far out of reach even for the most modern colliders. 


\subsection{Regge Trajectories}

The model presented here also provides a very simple explanation of Regge trajectories [10]. We have seen that the eigenvalue $\kappa_{P}$ of a particle is related to the mass $m_{P}$ of the particle by the relation:

$$
\kappa_{P}=\left(m_{P}^{\text {bare }}\right)^{2} / K^{2} .
$$

Let us gather $n_{f}$ fermions. The stability of the cluster is generally weak and the cluster, called a resonance $(R)$, decays rapidly. The spins of the individual fermions adds up to a maximum spin $j=n_{f} \hbar / 2$. Since the links between the individual fermions are weak their eigenvalues $\kappa_{P}$ approximately add up and

$$
\kappa_{R}=M_{R}^{2} / K^{2}=n_{f} \kappa_{P}=n_{f} m_{R}^{2} / K^{2}
$$

that is

$$
\left(M_{R}\right)^{2} \cong n_{f}\left(m_{P}\right)^{2}
$$

Finally

$$
j \cong\left(\hbar / 2\left(m_{P}\right)^{2}\right)\left(M_{R}\right)^{2} .
$$

This linear dependence of the spin $j$ along the square of the mass $M_{R}$ is called a Regge trajectory.

\subsection{The CPT Theorem}

The parity symmetry $P$ is broken by weak interactions as we have seen. On can wonder whether the time inversion symmetry $T$, or the charge conjugation symmetry $C$ can also be broken. We do not know the answer but a very general property, the CPT theorem, a product of these three invariance shows that the product CPT must be always verified.

The vector

$$
\phi=\left(\begin{array}{l}
\varphi_{1} \\
\varphi_{2} \\
\varphi_{3} \\
\varphi_{4}
\end{array}\right),
$$

represents the state of a particle. The state $\bar{\phi}$ of the associated anti-particle is obtained from $\phi$ through a mirror transformation that is a sign change of one of its components but, as discussed above, all signs have to be simultaneously changed. Whence

$$
\bar{\phi}=\left(\begin{array}{l}
-\varphi_{1} \\
-\varphi_{2} \\
-\varphi_{3} \\
-\varphi_{4}
\end{array}\right)=\left(\begin{array}{llll}
-1 & & & \\
& -1 & & \\
& & -1 & \\
& & & -1
\end{array}\right)\left(\begin{array}{l}
\varphi_{1} \\
\varphi_{2} \\
\varphi_{3} \\
\varphi_{4}
\end{array}\right)=C \phi,
$$

$C$ is the charge conjugation operator.

In other respects the time reversal operator $T$ changes the sign of the time component $\varphi_{1}$ of $\phi$ that is

$$
T\left(\begin{array}{c}
\varphi_{1} \\
\varphi_{2} \\
\varphi_{3} \\
\varphi_{4}
\end{array}\right)=\left(\begin{array}{c}
-\varphi_{1} \\
\varphi_{2} \\
\varphi_{3} \\
\varphi_{4}
\end{array}\right), \quad T=\left(\begin{array}{cccc}
-1 & & & \\
& 1 & & \\
& & 1 & \\
& & & 1
\end{array}\right)
$$

Finally the operation of reversing the direction of one axis, say the $z$ direction, amounts to a reflection of 
space (a mirror symmetry operation) in any number of space dimensions, and in three space dimensions it is equivalent to reflecting all space coordinates, because one can add an additional rotation of 180 degrees in the $x-y$ plane to complete the transformation. Let $P$ be the operator that reflects all space coordinates. $P$ amounts to changing the signs of the three spatial components $\varphi_{2}, \varphi_{3}$ and $\varphi_{4}$.

$$
P\left(\begin{array}{l}
\varphi_{1} \\
\varphi_{2} \\
\varphi_{3} \\
\varphi_{4}
\end{array}\right)=\left(\begin{array}{c}
\varphi_{1} \\
-\varphi_{2} \\
-\varphi_{3} \\
-\varphi_{4}
\end{array}\right),
$$

that is

$$
P=\left(\begin{array}{llll}
1 & & & \\
& -1 & & \\
& & -1 & \\
& & & -1
\end{array}\right) .
$$

We see that

$$
\mathrm{CPT}=1^{(4)}=\left(\begin{array}{llll}
1 & & & \\
& 1 & & \\
& & 1 & \\
& & & 1
\end{array}\right) .
$$

a simple and straightforward derivation of the CPT theorem.

\subsection{On Electric Charges of Fermions}

The electric charge $Q_{f}$ of a given fermion, lepton or quark, is derived from the hypercharge $Y_{f}$ through the formula

$$
Q_{f}=1 / 2\left(Y_{f}+2 \sigma_{f}\right),
$$

where $\sigma_{f}$ is the isospin of the particle.

The three generators of $\mathrm{SU}(2)$ are the three Pauli matrices

$$
\sigma_{1}=\left(\begin{array}{ll}
0 & 1 \\
1 & 0
\end{array}\right), \quad \sigma_{2}=\left(\begin{array}{cc}
0 & \mathrm{i} \\
-\mathrm{i} & 0
\end{array}\right), \quad \sigma_{3}=\left(\begin{array}{cc}
1 & 0 \\
0 & -1
\end{array}\right) .
$$

The interaction Lagrangian between leptons and their gauge field writes [3]

$$
\Lambda_{\psi-A}=\sum_{\mu} \psi^{\mathrm{T} *}\left(\sum_{\alpha=1, \cdots, 3} \sigma_{\alpha} A_{\alpha}^{\mu}\right) \psi .
$$

The quantities $A_{\alpha}^{\mu}$ are gauge fields. To make this expression more symmetric, the 2-dimensional identity matrix

$$
\sigma_{0}=\left(\begin{array}{ll}
1 & 0 \\
0 & 1
\end{array}\right)
$$

is added (and substracted) to the list of Pauli matrices (as in GWS theory)

$$
\Lambda_{\psi-A}=\sum_{\mu} \psi^{\mathrm{T} *}\left[\left(\sum_{\alpha=1, \cdots, 3} \sigma_{\alpha} A_{\alpha}^{\mu}\right)-\sigma_{0} A_{0}^{\mu}\right] \psi .
$$


The amplitude of the hypercharge $Y_{\text {lepton }}$ is defined by the coefficient of the generator of group U(1) namely the coefficient of the unit matrix $\sigma_{0}$ and, therefore, $\left|Y_{\text {lepton }}\right|=1$. One chooses $Y_{\text {leptons }}=-1$. The isospin of the electron is $\sigma_{e l}=-1 / 2$. Therefore its electric charge is $Q_{e l}=-1$ (that is $Q_{e l}=-e$ ). The isospin of the neutrino is $\sigma_{v}=+1 / 2$. Therefore its electric charge is $Q_{v}=0$, that is the neutrino is neutral.

Let us now turn towards the electric charges of quarks. The eight generators of SU(3) are the eight Gell-Mann matrices

$$
\begin{array}{ll}
\tau_{1}=\left(\begin{array}{lll}
0 & 1 & 0 \\
1 & 0 & 0 \\
0 & 0 & 0
\end{array}\right), & \tau_{2}=\left(\begin{array}{ccc}
0 & 0 & 1 \\
0 & 0 & 0 \\
1 & 0 & 0
\end{array}\right), \quad \tau_{3}=\left(\begin{array}{lll}
0 & 0 & 0 \\
0 & 0 & 1 \\
0 & 1 & 0
\end{array}\right) \\
\tau_{4}=\left(\begin{array}{ccc}
0 & \mathrm{i} & 0 \\
-\mathrm{i} & 0 & 0 \\
0 & 0 & 0
\end{array}\right), \quad \tau_{5}=\left(\begin{array}{ccc}
0 & 0 & \mathrm{i} \\
0 & 0 & 0 \\
-\mathrm{i} & 0 & 0
\end{array}\right), \quad \tau_{6}=\left(\begin{array}{ccc}
0 & 0 & 0 \\
0 & 0 & \mathrm{i} \\
0 & -\mathrm{i} & 0
\end{array}\right) \\
\tau_{7}=\left(\begin{array}{ccc}
1 & 0 & 0 \\
0 & -1 & 0 \\
0 & 0 & 0
\end{array}\right), & \tau_{8}=\left(\begin{array}{ccc}
1 & 0 & 0 \\
0 & 1 & 0 \\
0 & 0 & -2
\end{array}\right) .
\end{array}
$$

To make the expression of the Lagrangian more symmetric the 3-dimensional identity matrix

$$
\tau_{0}=\left(\begin{array}{lll}
1 & 0 & 0 \\
0 & 1 & 0 \\
0 & 0 & 1
\end{array}\right)
$$

is added (and substracted) to the list of Gell-Mann matrices

$$
\Lambda_{\psi-A}=\sum_{\mu} \psi^{\mathrm{T} *}\left[\left(\sum_{\alpha=0, \cdots, 8} \tau_{\alpha} B_{\alpha}^{\mu}\right)-\tau_{0} B_{0}^{\mu}\right] \psi .
$$

In order to compare the electric charges of leptons and quarks it is necessary to establish an endomorphism between the set of the eight Gell-Mann matrices and the set of three Pauli matrices. This endomorphism is given by

$$
\begin{aligned}
& \tau_{1} ; \tau_{2} ; \tau_{3} \rightarrow \sigma_{1} \\
& \tau_{4} ; \tau_{5} ; \tau_{6} \rightarrow \sigma_{2} \\
& \tau_{0} ; \tau_{7} ; \tau_{8} \rightarrow \sigma_{3}
\end{aligned}
$$

in the main term and $\tau_{0} \rightarrow \sigma_{0}$ in the last term whence

$$
\Lambda_{\psi-A}=\sum_{\mu} \psi^{\mathrm{T} *} 3\left[\left(\sum_{\alpha=1, \cdots, 3} \sigma_{\alpha} B_{\alpha}^{\mu}\right)-\left(\sigma_{0} / 3\right) B_{0}^{\mu}\right] \psi .
$$

One observes that $\left|Y_{\text {quark }}\right|=1 / 3$. One chooses $Y_{\text {quark }}=1 / 3$. The isospin of the up quark is $\sigma_{\text {up }}=+1 / 2$. Therefore the electric charge of the up quark is

$$
Q_{\text {up }}=2 / 3 \text { (that is } Q_{\text {up }}=2 / 3 e \text { ). }
$$

The isospin of the down quark is $\sigma_{\text {down }}=-1 / 2$. Therefore its electric charge is

$$
\left.Q_{\text {down }}=-1 / 3 \text { (that is } Q_{\text {down }}=-1 / 3 e\right) \text {. }
$$

\subsection{Three Families of Particles}

As already argued, the symmetry properties of bare particles are determined by the matrix $G_{P}$. The particles are 
created in high energy collisions experiments. In this type of experiments much energy is put down inside the core of a world point to such an amount that the value of cosmic temperature $b^{-1}$ inside the core could be severely modified and, thereby, could modify its geometrical properties. We propose to look for the origin of families of fermions in these cosmic temperature perturbations.

We have seen, in [1], that the dimensionality $d$ of space-time is given by $d=\operatorname{Int}(J b)$, where $J$ is the binary interaction between the cosmic bits and $b$ is a measure of cosmic bits disorder that is of cosmic temperature. The spatial dimension is $d_{S}=d-1$. A decrease of the cosmic temperature $b^{-1}$ results in an increase of the dimensionality $d$. When $b^{-1}$ decreases, the dimensionality of the core of the point of collision undergoes a series of changes.

1) $1>b J>0: d=0$. The core of a world point is very "hot" and dimensionless.

2) $2>b J>1: \quad d=1$. The core has one time dimension and no space dimensions.

3) $3>b J>2: d=2$. The core has one space dimension and one time dimension.

4) $4>b J>3: d=3$. The core has two space dimensions and one time dimension.

5) $5>b J>4: d=4$. This is the case for the ordinary space-time. The core has then three space dimensions and one time dimension.

The three domains $(3,4$ and 5$)$ of cosmic temperatures would correspond to the three families of particles. The domain (5) $\left(d_{s}=3\right)$ would be the domain of the electron family, the domain (4) $\left(d_{s}=2\right)$ that of the muon family and the domain (3) $\left(d_{S}=1\right)$ that of the tau family. There is no room for any other family. There are three families of fermions because there are three space dimensions in our space-time.

\subsection{On the CKM Matrix}

The idea that the three-fold multiplicity of families of particles may be explained by a dimensional re-organization of space-time in the core of a particle is supported by the following analysis of the CKM (Cabbibo, Kobayashi and Maskawa) matrix.

The CKM matrix $V$ describes the amplitudes of transition probabilities between the set of three quarks with isospin $-1 / 2$, namely $u, c$ and $t$, and the set of three quarks $d$, $s$ and $b$, with isospin $+1 / 2$ through weak interaction. The CKM matrix $V$ writes

$$
V=\left(\begin{array}{ccc}
V_{u d} & V_{u s} & V_{u b} \\
V_{c d} & V_{c s} & V_{c b} \\
V_{t d} & V_{t s} & V_{t b}
\end{array}\right) .
$$

The experimental values of the elements of the CKM matrix are given below in Equation (15).

Here we strive to find the CKM matrix by using a very simple geometrical argument. The probability $W_{\alpha \beta}=\left|V_{\alpha \beta}\right|^{2}$ of a transition of quark $\alpha$ into quark $\beta$ is assumed to be proportional to the overlap between quark $\alpha$ and quark $\beta$, that is by the relative number of world points that are common to $\alpha$ and $\beta$.

The perturbation $\gamma_{d_{S}}(r)$ in the vicinity of a particle seed that is in the core, in a $d$-dimensional space behaves as

$$
\gamma_{d_{S}}(r) \cong\left(r / \rho l^{*}\right)^{2-d_{S}}
$$

with $d_{s}=d-1 . \rho l^{*}$, the dimension of bare particles, that is the size of the core, is a standard of length. Let $r g(d)$ be the range of a $d$-dimensional bare particle. This size is the distance where $\gamma_{d_{s}}(r=r g(d))=1$.

For 3-dimensional quarks $\left(u\right.$ and $d$ ) one has $\gamma_{3}(r) \cong \rho l^{*} / r$. Then $1=\rho l^{*} / r g(3)$ and therefore $r g(3)=\rho l^{*}$. The number $n_{W}(3)$ of world points gathered to build a 3-dimensional bare particle is

$$
n_{w}(3)=4 \pi / 3\left(\rho l^{*}\right)^{3} /\left(l^{*}\right)^{3}=4 \pi / 3 \rho^{3} .
$$

For 2-dimensional quarks (s and $c$ ) one has $\gamma_{2}(r) \cong \log \left(r / \rho l^{*}\right)$. Then

$$
1=\log (e)=\log \left(r g(2) / \rho l^{*}\right)
$$

and, therefore, $r g(2)=e \rho l^{*}$. The number $n_{W}(2)$ of world points in a 2-dimensional bare particle is 


$$
n_{w}(2)=4 \pi\left(e \rho l^{*}\right)^{2} /\left(l^{*}\right)^{2}=4 \pi e^{2} \rho^{2} .
$$

Finally for 1 -dimensional quarks $(t$ and $b)$ one has $\gamma_{1}(r) \cong r / \rho l^{*}$. Then $1=r g(1) / \rho l^{*}$ and, therefore, $r g(1)=\rho l^{*}$. The number $n_{W}(1)$ of world points gathered to build a 1-dimensional bare particle is

$$
n_{W}(1)=2 \rho l^{*} / l^{*}=2 \rho \text {. }
$$
is

According to our hypothesis, the probability $W_{\alpha \beta}=\left|V_{\alpha \beta}\right|^{2}$ of transition between particle $\alpha$ and particle $\beta$

$$
W_{\alpha \beta}=n_{W}(d(\beta)) / n_{W}(d(\alpha)) \text {. }
$$

That is

$$
\begin{gathered}
n_{W}(3) / n_{W}(1)=\left(4 / 3\left(\rho l^{*}\right)^{3}\right) / 2 \rho\left(l^{*}\right)^{3}=\frac{2}{3} \pi \rho^{2} \\
n_{W}(3) / n_{W}(2)=\left(4 \pi / 3\left(\rho l^{*}\right)^{3}\right) / 4 \pi e^{2} \rho^{2}\left(l^{*}\right)^{3}=\frac{1}{3 e^{2}} \rho
\end{gathered}
$$

and

$$
n_{W}(2) / n_{W}(1)=4 \pi e^{2} \rho^{2}\left(l^{*}\right)^{3} / 2 \rho\left(l^{*}\right)^{3}=2 \pi e^{2} \rho .
$$

The matrix $W$ then writes

$$
W(\rho)=\left(\begin{array}{ccc}
1 & 3 \frac{e^{2}}{\rho} & \frac{3}{2 \pi} \frac{1}{\rho^{2}} \\
3 \frac{e^{2}}{\rho} & 1 & \frac{1}{2 e^{2} \pi \rho} \\
\frac{3}{2 \pi} \frac{1}{\rho^{2}} & \frac{1}{2 e^{2} \pi \rho} & 1
\end{array}\right) .
$$

Since one must have $\sum_{\beta} W_{\alpha \beta}=1$ the probabilities are normalized

$$
W_{\alpha \beta} \rightarrow W_{\alpha \beta} / \sum_{\gamma} W_{\alpha \gamma}
$$

Finally the transition probability amplitude is given by $\left|V_{\alpha \beta}\right|=\left(W_{\alpha \beta}\right)^{1 / 2}$. To find the range parameter $\rho$ we use an optimal mean square deviation procedure. The quantity to be minimized is

$$
\chi^{2} .(\rho)=\sum_{\alpha=1, \cdots, 3 ; \beta=1, \cdots, 3}\left[1-\frac{|V|_{\alpha \beta}^{\exp }}{W_{\alpha \beta}^{1 / 2}(\rho)}\right]^{2} .
$$

One finds $\rho=410$. Then the computed CKM matrix writes:

$$
|V|=\left(\begin{array}{lll}
0.974 & 0.226 & 0.002 \\
0.226 & 0.974 & 0.007 \\
0.002 & 0.007 & 0.999
\end{array}\right),
$$

to be compared with the experimental matrix [10]

$$
|V|^{\exp }=\left(\begin{array}{lll}
0.974 & 0.225 & 0.004 \\
0.225 & 0.973 & 0.041 \\
0.009 & 0.040 & 0.999
\end{array}\right) .
$$


The agreement is not that satisfactory, especially for the transitions between the quarks of muon family and those of tau family. This is not surprising given the crudeness of the calculations. One may consider, however, that the general trend is in the right direction and that the approach brings a valuable support to the granular structure of space-time and to the thermodynamic interpretation of the nature of fermions families. With the value $\rho \cong 410$ the size of a bare particle is

$$
l^{b}=\rho \times l^{*}=410 \times 0.52 \times 10^{-21} \mathrm{~cm}=2.1 \times 10^{-19} \mathrm{~cm},
$$

perhaps not completely out of the reach of realizable colliders.

\section{Conclusions}

The details of the organization of particles along the Standard model, their spins, charges and masses have been recovered in the framework of the discrete spaces model that we put forward. The neutrino chirality has been explained without appealing to a dichotomy between left and right universes. The introduction of this dichotomy is, in our opinion, a price too high to pay.

Our approach of physical phenomena implies a number of consequences:

1) The concept of infinity does not belong to the realm of physics. Infinity would only be a creation of mathematicians. We know that the observable universe is finite and therefore the infinitely large cannot be observed. We assume that, likewise, the infinitely small cannot be observed either.

2) The set of experimental results accumulated so far is enough to build a comprehensive theory of natural phenomena.

3) Many physicists believe that we cannot understand the laws of nature without appealing to complicated mathematics. Some physicists even believe that the necessary mathematics must be so complicated that they are outside the reach of a human brain. We do not share this prejudice. Notions of linear algebra and elements of group theory seem to be enough.

Finally let us list the main notions that have been introduced and that could be open to experimental observations.

a) First of all, we have introduced a metric limit $l^{*}$ (a cut-off) where both relativity and quantum mechanics loose their meanings. This solves the apparent incompatibility between these two theories. Exploring the metric limit implies energies of the order of $10^{4} \mathrm{TeV}$ outside the reach of currently available colliders. The size $\rho l^{*}$ of bare particles, however, could correspond to sizes of the order of $10^{2} \mathrm{TeV}$ that is of the order of $10^{-20} \mathrm{~cm}$. Very large linear colliders, using the CLIC technology for example, could perhaps explore that range of energy.

b) We have defined a so-called cosmic noise, a sort of temperature that describes the disorder of the most elementary physical systems (the cosmic bits) at a Planck scale. The cosmic noise, in our model, plays a central role in the organization and understanding of all natural phenomena. This noise could possibly be observed through phenomena relating to dark matter [3] or, perhaps, to the properties of quasars. The maps of dark matter would be, in reality, maps of cosmic noise.

c) The model has no need of a dichotomy of the universe between a right and a left universe. The left handedness character of a neutrino is a consequence of the present theory and no Higgs mechanism is necessary to give a mass to particles. The mass of the Higgs particle, if any, would be of the order of $10^{4} \mathrm{TeV}$.

d) The model opens the possibility to the existence of a non-standard family of particles whose main properties are described in Section 5.

Finally we, obviously, do not claim that the model of discrete spaces could be a sort of Grand Unified Theory (GUT), but we simply hope that some significant steps have been made towards the solution of the sixth Hilbert's problem [11] [12], which is the expression of axioms of physics. We could also say that, since it gives a physical interpretation to so many natural phenomena, the model of discrete spaces that we put forward probably contains some elements of reality.

\section{Acknowledgements}

I would like to express my best thanks to two institutions and to their managers. The first is the French Atomic Commission (CEA) with Dr Jacques Chappert who accepted that I spend one year learning high energy physics as an ordinary student at the Marseilles-Luminy University. He also accepted that I work on the subject for so long a time with so few published material. The other is the National Scientific Research Centre (CNRS) with 
Dr Bart van Tiggelen and Franck Hekking who welcomed me as an external researcher in their laboratory. Finally I thank my friend Pr Roger Maynard, my daughter-in-law Dr Ana Cabral and, obviously, my wife Michèle who incited me not to give up.

\section{References}

[1] Peretto, P. (2014) Journal of Modern Physics, 8, 563-575.

[2] Peretto, P. (2014) Journal of Modern Physics, 14, 1370-1386.

[3] Peretto, P. (2014) Journal of Modern Physics, 18, 2049-2062.

[4] Koide, Y. (1983) Physical Review D, 28, 252. http://dx.doi.org/10.1103/PhysRevD.28.252

[5] Dodelson, S., Melchiorri, A. and Slosar, A. (2006) Physical Review Letters, 97, Article ID: 04301.

[6] Aguilar, A. (2010) Physical Review D, 81, 098.

[7] Testa, M. (1975) Physics Letters B, 56, 53-56.

[8] Davies, C. (2013) Physical Review D, 81, 1103.

[9] Daum, M., et al. (2000) Physical Review Letters, 85, 1815.

[10] Collins, P.D.B. (1997) An Introduction to Regge Physics and High Energy Physics. Cambridge University Press, Cambridge.

[11] Nakamura, K., et al. (2010) Journal of Physics G: Nuclear and Particle Physics, 37, Article ID: 075012. http://dx.doi.org/10.1088/0954-3899/37/7A/075021

[12] Corry, L. (1994) Archives of History of Exact Sciences, 51, 83-168. http://dx.doi.org/10.1007/BF00375141 